\title{
MONOTONICITY AND COMPLETE MONOTONICITY OF TWO FUNCTIONS CONSTITUTED VIA THREE DERIVATIVES OF A FUNCTION INVOLVING TRIGAMMA FUNCTION
}

\author{
FENG QI
}

Dedicated to people facing and battling COVID-19

\begin{abstract}
In the paper, by convolution theorem of the Laplace transforms, a monotonicity rule for the ratio of two Laplace transforms, Bernstein's theorem

for completely monotonic functions, and other analytic techniques, the author

(1) presents the decreasing monotonicity of a ratio constituted via three derivatives of a function involving trigamma function;

(2) discovers necessary and sufficient conditions for a function constituted via three derivatives of a function involving trigamma function to be completely monotonic.

These results conform previous guesses posed by the author.
\end{abstract}

\section{Contents}

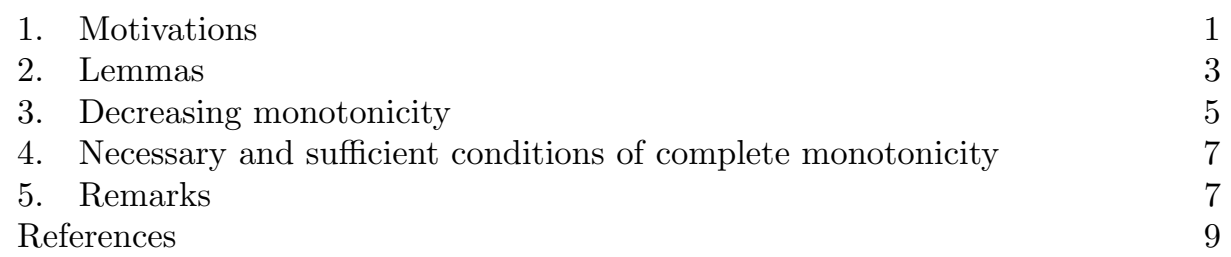

\section{Motivations}

In the literature [1, Section 6.4], the function

$$
\Gamma(z)=\int_{0}^{\infty} t^{z-1} e^{-t} \mathrm{~d} t, \quad \Re(z)>0
$$

and its logarithmic derivative $\psi(z)=[\ln \Gamma(z)]^{\prime}=\frac{\Gamma^{\prime}(z)}{\Gamma(z)}$ are respectively called Euler's gamma function and digamma function. Further, the functions $\psi^{\prime}(z)$, $\psi^{\prime \prime}(z), \psi^{\prime \prime \prime}(z)$, and $\psi^{(4)}(z)$ are known as trigamma, tetragamma, pentagamma, and hexagamma functions respectively. As a whole, all the derivatives $\psi^{(k)}(z)$ for $k \in\{0\} \cup \mathbb{N}$ are known as polygamma functions, where $\mathbb{N}$ denotes the set of all positive integers.

Recall from Chapter XIII in [3, Chapter 1 in 12, and Chapter IV in 13 that, if a function $f(x)$ on an interval $I$ has derivatives of all orders on $I$ and satisfies

2010 Mathematics Subject Classification. Primary 33B15; Secondary 26A48, 26A51, 26D07, 33B10, 44A10, 44A35.

Key words and phrases. monotonicity; complete monotonicity; trigamma function; derivative; difference; ratio; Laplace transform; convolution theorem; monotonicity rule; Bernstein's theorem; exponential function; inequality; guess.

This paper was typeset using $\mathcal{A} \mathcal{M} \mathcal{S}$-LATEX. 
$(-1)^{n} f^{(n)}(x) \geq 0$ for $x \in I$ and $n \in\{0\} \cup \mathbb{N}$, then we call $f(x)$ a completely monotonic function on $I$.

There are a number of papers and mathematicians dedicated to investigation of complete monotonicity of some functions involving the gamma and polygamma functions. For more information and details, please refer to the papers [2, 9, 11, 15] and closely related references therein.

Let

$$
G(x)=x\left[x \psi^{\prime}(x)-1\right]-\frac{1}{2}=x^{2}\left[\psi^{\prime}(x)-\frac{1}{x}-\frac{1}{2 x^{2}}\right], \quad x \in(0, \infty) .
$$

It is easy to obtain that

$$
G^{\prime}(x)=x^{2} \psi^{\prime \prime}(x)+2 x \psi^{\prime}(x)-1
$$

and

$$
G^{(\ell)}(x)=x^{2} \psi^{(\ell+1)}(x)+2 \ell x \psi^{(\ell)}(x)+\ell(\ell-1) \psi^{(\ell-1)}(x)
$$

for $\ell \geq 2$. For $k \in\{0\} \cup \mathbb{N}$ and $\theta_{k}, \tau_{k} \in \mathbb{R}$, let

$$
\mathcal{G}_{k, \theta_{k}}(x)=G^{(2 k+1)}(x)+\theta_{k}\left[G^{(k)}(x)\right]^{2}
$$

and

$$
\mathfrak{G}_{k, \tau_{k}}(x)=\frac{G^{(2 k+1)}(x)}{\left[(-1)^{k} G^{(k)}(x)\right]^{\tau_{k}}}
$$

on $(0, \infty)$. In [7, Theorem 3.1 and Theorem 4.1], the author discovered the following necessary and sufficient conditions, limits, and double inequality:

(1) if and only if $\theta_{k} \geq \frac{3(2 k+2) !}{k !(k+1) !}$, the function $\mathcal{G}_{k, \theta_{k}}(x)$ is completely monotonic on $(0, \infty)$;

(2) if and only if $\theta_{k} \leq 0$, the function $-\mathcal{G}_{k, \theta_{k}}(x)$ is completely monotonic on $(0, \infty)$;

(3) if and only if $\tau_{k} \geq 2$, the function $\mathfrak{G}_{k, \tau_{k}}(x)$ is decreasing on $(0, \infty)$;

(4) if $\tau_{k} \leq 1$, the function $\mathfrak{G}_{k, \tau_{k}}(x)$ is increasing on $(0, \infty)$;

(5) only if

$$
\tau_{k} \leq \begin{cases}\psi^{\prime}(1), & k=0 \\ -\frac{\psi^{\prime \prime \prime}(1)}{\psi^{\prime}(1) \psi^{\prime \prime}(1)}, & k=1 \\ \frac{k-1}{k} \frac{\psi^{(k-1)}(1) \psi^{(2 k+1)}(1)}{\psi^{(k)}(1) \psi^{(2 k)}(1)}, & k \geq 2,\end{cases}
$$

the function $\mathfrak{G}_{k, \tau_{k}}(x)$ is increasing on $(0, \infty)$;

(6) the limits

$$
\lim _{x \rightarrow 0^{+}} \mathfrak{G}_{k, \tau_{k}}(x)= \begin{cases}-2^{\tau_{0}}, & k=0 \\ 6 \psi^{\prime \prime}(1), & k=1 \\ \frac{2(2 k+1)}{(k-1)^{\tau_{k}} k^{\tau_{k}-1}} \frac{\psi^{(2 k)}(1)}{\left|\psi^{(k-1)}(1)\right|}, & k \geq 2\end{cases}
$$

and

$$
\lim _{x \rightarrow \infty} \mathfrak{G}_{k, \tau_{k}}(x)= \begin{cases}-\infty, & \tau_{k}>2 \\ -\frac{3(2 k+2) !}{k !(k+1) !}, & \tau_{k}=2 \\ 0, & \tau_{k}<2\end{cases}
$$

are valid; 
(7) the double inequality

$$
-\frac{3(2 k+2) !}{k !(k+1) !}<\mathfrak{G}_{k, 2}(x)< \begin{cases}-4, & k=0 \\ 6 \psi^{\prime \prime}(1), & k=1 \\ \frac{2(2 k+1)}{(k-1)^{2} k} \frac{\psi^{(2 k)}(1)}{\left|\psi^{(k-1)}(1)\right|}, & k \geq 2\end{cases}
$$

is valid on $(0, \infty)$ and sharp in the sense that the lower and upper bounds cannot be replaced by any greater and less numbers respectively.

In [7, Remark 3.1], basing on the proof of necessary conditions in [7, Theorem 3.1], the author guessed that the function

$$
\mathscr{G}_{k}(x)=\frac{G^{(2 k+2)}(x)}{G^{(k)}(x) G^{(k+1)}(x)}, \quad k \in\{0\} \cup \mathbb{N}
$$

should be decreasing on $(0, \infty)$ and that the double inequality

$$
-\frac{6(2 k+2) !}{k !(k+1) !}<\mathscr{G}_{k}(x)< \begin{cases}-4 \psi^{\prime}(1), & k=0 \\ -\frac{6 \psi^{\prime \prime \prime}(1)}{\psi^{\prime}(1)}, & k=1 \\ \frac{2(2 k+1)}{k^{2}(k-1)} \frac{\psi^{(2 k+1)}(1)}{\psi^{(k-1)}(1) \psi^{(k)}(1)}, & k \geq 2\end{cases}
$$

should be valid and sharp in the sense that the lower and upper bounds cannot be replaced by any bigger and small numbers.

For $m, n \in\{0\} \cup \mathbb{N}$, let

$$
\mathscr{G}_{m, n}(x)=\frac{G^{(m+n+1)}(x)}{G^{(m)}(x) G^{(n)}(x)}
$$

and

$$
\mathscr{G}_{m, n ; \lambda_{m, n}}(x)=G^{(m+n+1)}(x)+\lambda_{m, n} G^{(m)}(x) G^{(n)}(x)
$$

on $(0, \infty)$. It is clear that

$$
\begin{gathered}
\mathscr{G}_{m, n}(x)=\mathscr{G}_{n, m}(x), \quad \mathscr{G}_{m, n ; \lambda_{m, n}}(x)=\mathscr{G}_{n, m ; \lambda_{n, m}}(x), \\
\mathscr{G}_{k, k+1}(x)=\mathscr{G}_{k}(x), \quad \mathscr{G}_{k, k}(x)=\mathfrak{G}_{k, \tau_{k}}(x), \quad \mathscr{G}_{k, k ; \lambda_{k, k}}(x)=\mathcal{G}_{k, \theta_{k}}(x)
\end{gathered}
$$

with $\tau_{k}=2$ and $\lambda_{k, k}=\theta_{k}$. This means that the functions $\mathscr{G}_{m, n}(x)$ and $\mathscr{G}_{m, n ; \lambda_{m, n}}(x)$ are generalizations of the ones $\mathcal{G}_{k, \theta_{k}}(x)$ and $\mathfrak{G}_{k, \tau_{k}}(x)$ defined in (1.1) and (1.2).

In this paper, we mainly

(1) prove that the function $\mathscr{G}_{m, n}(x)$ for $m, n \in\{0\} \cup \mathbb{N}$ is decreasing on $(0, \infty)$;

(2) discover necessary and sufficient conditions on $\lambda_{m, n}$ such that the functions $\pm(-1)^{m+n+1} \mathscr{G}_{m, n ; \lambda_{m, n}}(x)$ are completely monotonic on $(0, \infty)$.

The decreasing monotonicity of the function $\mathscr{G}_{m, n}(x)$ for $m, n \in\{0\} \cup \mathbb{N}$ on $(0, \infty)$ confirms that the above guesses posed by the author in [7, Remark 3.1] are true.

\section{LEMMAS}

The following lemmas are necessary in this paper.

Lemma 2.1 (Convolution theorem for the Laplace transforms [13, pp. 91-92]). Let $f_{k}(t)$ for $k=1,2$ be piecewise continuous in arbitrary finite intervals included in $(0, \infty)$. If there exist some constants $M_{k}>0$ and $c_{k} \geq 0$ such that $\left|f_{k}(t)\right| \leq M_{k} e^{c_{k} t}$ for $k=1,2$, then

$$
\int_{0}^{\infty}\left[\int_{0}^{t} f_{1}(u) f_{2}(t-u) \mathrm{d} u\right] e^{-s t} \mathrm{~d} t=\int_{0}^{\infty} f_{1}(u) e^{-s u} \mathrm{~d} u \int_{0}^{\infty} f_{2}(v) e^{-s v} \mathrm{~d} v .
$$


Lemma 2.2 ([14, Lemma 4]). Let the functions $A(t)$ and $B(t) \neq 0$ be defined on $(0, \infty)$ such that their Laplace transforms $\int_{0}^{\infty} A(t) e^{-x t} \mathrm{~d} t$ and $\int_{0}^{\infty} B(t) e^{-x t} \mathrm{~d} t$ exist. If the ratio $\frac{A(t)}{B(t)}$ is increasing, then the ratio $\frac{\int_{0}^{\infty} A(t) e^{-x t} \mathrm{~d} t}{\int_{0}^{\infty} B(t) e^{-x t} \mathrm{~d} t}$ is decreasing on $(0, \infty)$.

Lemma 2.3. Let

$$
w(t)= \begin{cases}\frac{e^{t}\left[(t-2) e^{t}+t+2\right]}{\left(e^{t}-1\right)^{3}}, & t \neq 0 \\ \frac{1}{6}, & t=0\end{cases}
$$

Then the following conclusions are valid:

(1) the function $w(t)$ is infinitely differentiable, positive, and even on $(-\infty, \infty)$, is increasing on $(-\infty, 0)$, and is decreasing on $(0, \infty)$;

(2) the function $w(t)$ is logarithmically concave on $(-\infty, \infty)$;

(3) for fixed $s \in(0,1)$, the ratio $\frac{w^{s}(t)}{w(s t)}$ is even in $t \in(-\infty, \infty)$ and decreasing in $t$ from $(0, \infty)$ onto $\left(0,6^{1-s}\right)$.

Proof. The infinite differentiability, positivity, even property, monotonicity, and logarithmic concavity of the function $w(t)$ on $(-\infty, \infty)$ have been proved in [7, Lemma 2.1].

Since the function $w(t)$ is even on $(-\infty, \infty)$, it is immediate that the ratio $\frac{w^{s}(t)}{w(s t)}$ is even on $(-\infty, \infty)$.

It is straightforward that

$$
\lim _{t \rightarrow 0} \frac{w^{s}(t)}{w(s t)}=\frac{\lim _{t \rightarrow 0} w^{s}(t)}{\lim _{t \rightarrow 0} w(s t)}=\frac{(1 / 6)^{s}}{1 / 6}=6^{1-s}
$$

and

$$
\lim _{t \rightarrow \infty} \frac{w^{s}(t)}{w(s t)}=\frac{\lim _{t \rightarrow \infty}\left[e^{t} w(t) / t\right]^{s}}{\lim _{t \rightarrow \infty}\left[e^{s t} w(s t) / s t\right]} \lim _{t \rightarrow \infty} \frac{e^{s t} / s t}{\left(e^{t} / t\right)^{s}}=0,
$$

where we used the $\operatorname{limit}_{t \rightarrow \infty} \frac{e^{t} w(t)}{t}=1$.

The first derivative of the ratio $\frac{w^{s}(t)}{w(s t)}$ is

$$
\frac{\mathrm{d}}{\mathrm{d} t}\left[\frac{w^{s}(t)}{w(s t)}\right]=\frac{s w^{s}(t)}{w(s t)}\left[\frac{w^{\prime}(t)}{w(t)}-\frac{w^{\prime}(s t)}{w(s t)}\right] .
$$

Accordingly, for obtaining monotonicity of the ratio $\frac{w^{s}(t)}{w(s t)}$, it is sufficient to show that the ratio $\frac{w^{\prime}(t)}{w(t)}$ is decreasing on $(0, \infty)$. For this, it is sufficient to show that the function $w(t)$ is logarithmically concave on $(0, \infty)$. The proof of Lemma 2.3 is complete.

Lemma 2.4 ([7, Lemma 2.2]). For $k \in\{0\} \cup \mathbb{N}$, the function $(-1)^{k} G^{(k)}(x)$ is completely monotonic on $(0, \infty)$, with the limits

$$
\lim _{x \rightarrow 0^{+}}\left[(-1)^{k} G^{(k)}(x)\right]= \begin{cases}\frac{1}{2}, & k=0 \\ 1, & k=1 \\ (-1)^{k} k(k-1) \psi^{(k-1)}(1), & k \geq 2\end{cases}
$$

and

$$
\lim _{x \rightarrow \infty}\left[(-1)^{k} x^{k+1} G^{(k)}(x)\right]=\frac{k !}{6} .
$$


Lemma 2.5 (Bernstein's theorem [13, p. 161, Theorem 12b]). A function $f(x)$ is completely monotonic on $(0, \infty)$ if and only if

$$
f(x)=\int_{0}^{\infty} e^{-x t} \mathrm{~d} \sigma(t), \quad x \in(0, \infty),
$$

where $\sigma(s)$ is non-decreasing and the integral in $(2.3)$ converges for $x \in(0, \infty)$.

\section{Decreasing monotonicity}

In this section, we prove that the function $\mathscr{G}_{m, n}(x)$ defined in 1.4$)$ is decreasing.

Theorem 3.1. For $m, n \in\{0\} \cup \mathbb{N}$, the function $\mathscr{G}_{m, n}(x)$ in 1.4 is decreasing in $x \in(0, \infty)$ and maps from $(0, \infty)$,

(1) if $(m, n)=(0,0)$, onto the interval $(-6,-4)$;

(2) if $(m, n) \in\{(1,0),(0,1)\}$, onto the interval $\left(-12,-4 \psi^{\prime}(1)\right)$;

(3) if $(m, n) \in\{(2,0),(0,2)\}$, onto the interval $\left(-18, \frac{6 \psi^{\prime \prime}(1)}{\psi^{\prime}(1)}\right)$;

(4) if $(m, n)=(1,1)$, onto the interval $\left(-36,6 \psi^{\prime \prime}(1)\right)$;

(5) if $(m, n) \in\{(2,1),(1,2)\}$, onto the interval $\left(-72,-\frac{6 \psi^{\prime \prime \prime}(1)}{\psi^{\prime}(1)}\right)$;

(6) if $m, n \geq 2$, onto the interval

$$
\left(-\frac{6(m+n+1) !}{m ! n !}, \frac{(m+n+1)(m+n)}{m n(m-1)(n-1)} \frac{\psi^{(m+n)}(1)}{\psi^{(m-1)}(1) \psi^{(n-1)}(1)}\right) .
$$

Consequently, for $m, n \in\{0\} \cup \mathbb{N}$, the double inequality

$$
\begin{aligned}
& -\frac{6(m+n+1) !}{m ! n !}<\mathscr{G}_{m, n}(x) \\
& \quad< \begin{cases}-4, & (m, n)=(0,0) \\
-4 \psi^{\prime}(1), & (m, n) \in\{(1,0),(0,1)\} \\
\frac{6 \psi^{\prime \prime}(1)}{\psi^{\prime}(1)} & (m, n) \in\{(2,0),(0,2)\} \\
6 \psi^{\prime \prime}(1), & (m, n)=(1,1) \\
-\frac{6 \psi^{\prime \prime \prime}(1)}{\psi^{\prime}(1)}, & (m, n) \in\{(2,1),(1,2)\} \\
\frac{(m+n+1)(m+n)}{m n(m-1)(n-1)} \frac{\psi^{(m+n)}(1)}{\psi^{(m-1)}(1) \psi^{(n-1)}(1)}, & m, n \geq 2\end{cases}
\end{aligned}
$$

is valid on $(0, \infty)$ and sharp in the sense that the lower and upper bounds cannot be replaced by any larger and smaller numbers respectively.

Proof. Since $\mathscr{G}_{m, n}(x)=\mathscr{G}_{n, m}(x)$, for convenience, without loss of generality, we assume $m \geq n \geq 0$ in this proof.

In the proof of [8, Theorem 4], the author derived an integral representation

$$
G(x)=\int_{0}^{\infty} w(t) e^{-x t} \mathrm{~d} t .
$$

Then the function $\mathscr{G}_{m, n}(x)$ can be reformulated as

$$
\begin{aligned}
\mathscr{G}_{m, n}(x) & =-\frac{\int_{0}^{\infty} t^{m+n+1} w(t) e^{-x t} \mathrm{~d} t}{\int_{0}^{\infty} t^{m} w(t) e^{-x t} \mathrm{~d} t \int_{0}^{\infty} t^{n} w(t) e^{-x t} \mathrm{~d} t} \\
& =-\frac{\int_{0}^{\infty} t^{m+n+1} w(t) e^{-x t} \mathrm{~d} t}{\int_{0}^{\infty}\left[\int_{0}^{t} u^{m}(t-u)^{n} w(u) w(t-u) \mathrm{d} u\right] e^{-x t} \mathrm{~d} t}
\end{aligned}
$$


where we used Lemma 2.1. Further, by Lemma 2.2 it is sufficient to prove that the ratio

$$
\begin{aligned}
W_{m, n}(t) & =\frac{t^{m+n+1} w(t)}{\int_{0}^{t} u^{m}(t-u)^{n} w(u) w(t-u) \mathrm{d} u} \\
& =\frac{w(t)}{\int_{0}^{1} s^{m}(1-s)^{n} w(s t) w((1-s) t) \mathrm{d} s} \\
& =\frac{1}{\int_{0}^{1} s^{m}(1-s)^{n} \frac{w(s t)}{w^{s}(t)} \frac{w((1-s) t)}{w^{1-s}(t)} \mathrm{d} s}
\end{aligned}
$$

is decreasing on $(0, \infty)$, where we changed the variable $u=s t$. For this, it is sufficient to verify that the ratio $\frac{w^{s}(t)}{w(s t)}$ is decreasing in $t \in(0, \infty)$ for fixed $s \in(0,1)$. This requirement has been verified in Lemma 2.3 . As a result, the function $\mathscr{G}_{m, n}(x)$ is decreasing on $(0, \infty)$.

Making use of the limits (2.1) and (2.2) in Lemma 2.4 yields

$$
\begin{aligned}
& \lim _{x \rightarrow 0^{+}} \mathscr{G}_{m, n}(x)=\frac{\lim _{x \rightarrow 0^{+}} G^{(m+n+1)}(x)}{\lim _{x \rightarrow 0^{+}} G^{(m)}(x) \lim _{x \rightarrow 0^{+}} G^{(n)}(x)} \\
& = \begin{cases}\frac{\lim _{x \rightarrow 0^{+}} G^{\prime}(x)}{\lim _{x \rightarrow 0^{+}} G(x) \lim _{x \rightarrow 0^{+}} G(x)}, & (m, n)=(0,0) \\
\frac{\lim _{x \rightarrow 0^{+}} G^{\prime \prime}(x)}{\lim _{x \rightarrow 0^{+}} G^{\prime}(x) \lim _{x \rightarrow 0^{+}} G(x)}, & (m, n)=(1,0) \\
\frac{\lim _{x \rightarrow 0^{+}} G^{\prime \prime \prime}(x)}{\lim _{x \rightarrow 0^{+}} G^{\prime \prime}(x) \lim _{x \rightarrow 0^{+}} G(x)}, & (m, n)=(2,0) \\
\frac{\lim _{x \rightarrow 0^{+}} G^{\prime \prime \prime}(x)}{\lim _{x \rightarrow 0^{+}} G^{\prime}(x) \lim _{x \rightarrow 0^{+}} G^{\prime}(x)}, & (m, n)=(1,1) \\
\frac{\lim _{x \rightarrow 0^{+}} G^{(4)}(x)}{\lim _{x \rightarrow 0^{+}} G^{\prime \prime}(x) \lim _{x \rightarrow 0^{+}} G^{\prime}(x)}, & (m, n)=(2,1) \\
\frac{\lim _{x \rightarrow 0^{+}} G^{(m+n+1)}(x)}{\lim _{x \rightarrow 0^{+}} G^{(m)}(x) \lim _{x \rightarrow 0^{+}} G^{(n)}(x)}, & m \geq n \geq 2\end{cases} \\
& = \begin{cases}-4, & (m, n)=(0,0) \\
-4 \psi^{\prime}(1), & (m, n)=(1,0) \\
\frac{6 \psi^{\prime \prime}(1)}{\psi^{\prime}(1)}, & (m, n)=(2,0) \\
6 \psi^{\prime \prime}(1), & (m, n)=(1,1) \\
-\frac{6 \psi^{\prime \prime \prime}(1)}{\psi^{\prime}(1)}, & (m, n)=(2,1) \\
\frac{(m+n+1)(m+n)}{m n(m-1)(n-1)} \frac{\psi^{(m+n)}(1)}{\psi^{(m-1)}(1) \psi^{(n-1)}(1)}, & m \geq n \geq 2\end{cases}
\end{aligned}
$$

and

$$
\begin{aligned}
\lim _{x \rightarrow \infty} \mathscr{G}_{m, n}(x) & =-\frac{\lim _{x \rightarrow \infty}\left[(-1)^{m+n+1} x^{m+n+2} G^{(m+n+1)}(x)\right]}{\lim _{x \rightarrow \infty}\left[(-1)^{m} x^{m+1} G^{(m)}(x)\right] \lim _{x \rightarrow \infty}\left[(-1)^{n} x^{n+1} G^{(n)}(x)\right]} \\
& =-\frac{6(m+n+1) !}{m ! n !} .
\end{aligned}
$$

Therefore, those "onto" properties and the double inequality (3.1) and its sharpness follow readily. The proof of Theorem 3.1 is complete. 


\section{NeCESSARY AND SUFFICIENT CONDITIONS OF COMPLETE MONOTONICITY}

In this section, we discover necessary and sufficient conditions on $\lambda_{m, n}$ such that the functions $\pm(-1)^{m+n+1} \mathscr{G}_{m, n ; \lambda_{m, n}}(x)$ defined in 1.5 are completely monotonic.

Theorem 4.1. For $m, n \in\{0\} \cup \mathbb{N}$,

(1) if and only if $\lambda_{m, n} \leq 0$, the function $(-1)^{m+n+1} \mathscr{G}_{m, n ; \lambda_{m, n}}(x)$ is completely monotonic on $(0, \infty)$;

(2) if and only if $\lambda_{m, n} \geq \frac{6(m+n+1) !}{m ! n !}$, the function $(-1)^{m+n} \mathscr{G}_{m, n ; \lambda_{m, n}}(x)$ is completely monotonic on $(0, \infty)$.

Proof. By the integral representation $(3.2)$, we can write $\mathscr{G}_{m, n ; \lambda_{m, n}}(x)$ in 1.5 as

$$
\begin{aligned}
\mathscr{G}_{m, n ; \lambda_{m, n}}(x)= & (-1)^{m+n+1}\left[\int_{0}^{\infty} t^{m+n+1} w(t) e^{-x t} \mathrm{~d} t\right. \\
& \left.-\lambda_{m, n} \int_{0}^{\infty} t^{m} w(t) e^{-x t} \mathrm{~d} t \int_{0}^{\infty} t^{n} w(t) e^{-x t} \mathrm{~d} t\right] .
\end{aligned}
$$

Utilizing Lemma 2.1 results in

$$
\begin{gathered}
(-1)^{m+n+1} \mathscr{G}_{m, n ; \lambda_{m, n}}(x) \\
=\int_{0}^{\infty} t^{m+n+1} w(t) e^{-x t} \mathrm{~d} t-\lambda_{m, n} \int_{0}^{\infty}\left[\int_{0}^{t} u^{m}(t-u)^{n} w(u) w(t-u) \mathrm{d} u\right] e^{-x t} \mathrm{~d} t \\
=\int_{0}^{\infty}\left[t^{m+n+1} w(t)-\lambda_{m, n} \int_{0}^{t} u^{m}(t-u)^{n} w(u) w(t-u) \mathrm{d} u\right] e^{-x t} \mathrm{~d} t \\
=\int_{0}^{\infty}\left[1-\lambda_{m, n} \frac{\int_{0}^{t} u^{m}(t-u)^{n} w(u) w(t-u) \mathrm{d} u}{t^{m+n+1} w(t)}\right] t^{m+n+1} w(t) e^{-x t} \mathrm{~d} t \\
=\int_{0}^{\infty}\left[1-\frac{\lambda_{m, n}}{W_{m, n}(t)}\right] t^{m+n+1} w(t) e^{-x t} \mathrm{~d} t .
\end{gathered}
$$

In the proof of Theorem 3.1 in last section, the function $W_{m, n}(t)$ has been proved to be decreasing on $(0, \infty)$. Direct computation arrives at

$$
\begin{aligned}
& \lim _{t \rightarrow 0} W_{m, n}(t)=\frac{\lim _{t \rightarrow 0} w(t)}{\int_{0}^{1} s^{m}(1-s)^{n} \lim _{t \rightarrow 0}[w(s t) w((1-s) t)] \mathrm{d} s} \\
& =\frac{6}{\int_{0}^{1} s^{m}(1-s)^{n} \mathrm{~d} s}=\frac{6}{B(m+1, n+1)}=\frac{6(m+n+1) !}{m ! n !}
\end{aligned}
$$

and

$$
\begin{aligned}
\lim _{t \rightarrow \infty} W_{m, n}(t) & =\frac{\lim _{t \rightarrow \infty} \frac{e^{t} w(t)}{t}}{\int_{0}^{1} s^{m}(1-s)^{n} \lim _{t \rightarrow \infty} \frac{e^{s t} w(s t)}{s t} \lim _{t \rightarrow \infty} \frac{e^{(1-s) t} w((1-s) t)}{(1-s) t} \mathrm{~d} s} \lim _{t \rightarrow \infty} \frac{1}{s(1-s) t} \\
& =0
\end{aligned}
$$

where we used the $\operatorname{limit}_{t \rightarrow \infty} \frac{e^{t} w(t)}{t}=1$. Accordingly, by virtue of Lemma 2.5. if and only if $\lambda_{m, n} \leq 0$, the function $(-1)^{m+n+1} \mathscr{G}_{m, n ; \lambda_{m, n}}(x)$ is completely monotonic on $(0, \infty)$; if and only if $\lambda_{m, n} \geq \frac{6(m+n+1) !}{m ! n !}$, the function $(-1)^{m+n} \mathscr{G}_{m, n ; \lambda_{m, n}}(x)$ is completely monotonic on $(0, \infty)$. The proof of Theorem 4.1 is complete.

\section{REMARKS}

Finally, we list several remarks on our main results an their proofs.

Remark 5.1. The third conclusion in Lemma 2.3 in this paper generalizes a result in [7. Lemma 2.1]: the ratio $\frac{w(2 t)}{w^{2}(t)}$ is even on $(-\infty, \infty)$, increasing from $(-\infty, 0)$ onto $(0,6)$, and decreasing from $(0, \infty)$ onto $(0,6)$. 
Remark 5.2. The double inequality (3.1) is a generalization of the double inequality (1.3) which was guessed in [7, Remark 3.1] by the author.

Remark 5.3. For $m, n \in\{0\} \cup \mathbb{N}$, direct differentiation gives

$$
\mathscr{G}_{m, n}^{\prime}(x)=\frac{G^{(m+n+2)}(x)\left[G^{(m)}(x) G^{(n)}(x)\right]-G^{(m+n+1)}(x)\left[G^{(m)}(x) G^{(n)}(x)\right]^{\prime}}{\left[G^{(m)}(x) G^{(n)}(x)\right]^{2}}
$$

on $(0, \infty)$. The decreasing monotonicity of $\mathscr{G}_{m, n}(x)$ in Theorem 3.1 implies that, for $m, n \in\{0\} \cup \mathbb{N}$, the inequality

$$
G^{(m+n+1)}(x)\left[G^{(m)}(x) G^{(n)}(x)\right]^{\prime}-G^{(m+n+2)}(x)\left[G^{(m)}(x) G^{(n)}(x)\right]>0,
$$

equivalently,

$$
\frac{\left[G^{(m)}(x) G^{(n)}(x)\right]^{\prime}}{G^{(m)}(x) G^{(n)}(x)}<\frac{G^{(m+n+2)}(x)}{G^{(m+n+1)}(x)}
$$

is valid on $(0, \infty)$.

We guess that, for $m, n \in\{0\} \cup \mathbb{N}$, the function

$$
G^{(m+n+1)}(x)\left[G^{(m)}(x) G^{(n)}(x)\right]^{\prime}-G^{(m+n+2)}(x)\left[G^{(m)}(x) G^{(n)}(x)\right]
$$

is completely monotonic in $x \in(0, \infty)$.

One can also consider necessary and sufficient conditions on $\Upsilon_{m, n} \in \mathbb{R}$ for $m, n \in$ $\{0\} \cup \mathbb{N}$ such that the function

$$
G^{(m+n+1)}(x)\left[G^{(m)}(x) G^{(n)}(x)\right]^{\prime}-\Upsilon_{m, n} G^{(m+n+2)}(x)\left[G^{(m)}(x) G^{(n)}(x)\right]
$$

and its negativity are respectively completely monotonic on $(0, \infty)$.

Remark 5.4. Direct computation gives

$$
\begin{gathered}
\lim _{x \rightarrow 0^{+}} \frac{G^{(m+n+2)}(x)}{G^{(m+1)}(x) G^{(n)}(x)+G^{(m)}(x) G^{(n+1)}(x)} \\
=-\lim _{x \rightarrow 0^{+}} \frac{(-1)^{m+n+2} G^{(m+n+2)}(x)}{\left(\begin{array}{c}
{\left[(-1)^{m+1} G^{(m+1)}(x)\right]\left[(-1)^{n} G^{(n)}(x)\right]} \\
+\left[(-1)^{m} G^{(m)}(x)\right]\left[(-1)^{n+1} G^{(n+1)}(x)\right]
\end{array}\right)} \\
\lim _{x \rightarrow 0^{+}} \frac{(-1)^{2} G^{\prime \prime}(x)}{\left[-G^{\prime}(x)\right] G(x)+G(x)\left[(-1) G^{\prime}(x)\right]}, \quad(m, n)=(0,0) \\
\lim _{x \rightarrow 0^{+}} \frac{(-1)^{3} G^{\prime \prime \prime}(x)}{\left[(-1)^{2} G^{\prime \prime}(x)\right] G(x)+\left[-G^{\prime}(x)\right]\left[-G^{\prime}(x)\right]}, \quad(m, n) \in\{(1,0),(0,1)\} \\
\lim _{x \rightarrow 0^{+}} \frac{(-1)^{4} G^{(4)}(x)}{\left[(-1)^{3} G^{\prime \prime \prime}(x)\right] G(x)+G^{\prime \prime}(x)\left[-G^{\prime}(x)\right]}, \quad(m, n) \in\{(2,0),(0,2)\} \\
\lim _{x \rightarrow 0^{+}} \frac{(-1)^{4} G^{(4)}(x)}{\left[(-1)^{2} G^{\prime \prime}(x)\right]\left[-G^{\prime}(x)\right]+\left[-G^{\prime}(x)\right]\left[(-1)^{2} G^{\prime \prime}(x)\right]}, \quad(m)=(1,1) \\
\lim _{x \rightarrow 0^{+}} \frac{(-1)^{5} G^{(5)}(x)}{\left(\begin{array}{c}
{\left[(-1)^{3} G^{\prime \prime \prime}(x)\right]\left[-G^{\prime}(x)\right]} \\
+\left[(-1)^{2} G^{\prime \prime}(x)\right]\left[(-1)^{2} G^{\prime \prime}(x)\right]
\end{array}\right)} \\
\left.\lim _{x \rightarrow 0^{+}} \frac{(-1)^{m+n+2} G^{(m+n+2)}(x)}{\left(\begin{array}{c}
{\left[(-1)^{m+1} G^{(m+1)}(x)\right]\left[(-1)^{n} G^{(n)}(x)\right]} \\
+\left[(-1)^{m} G^{(m)}(x)\right]\left[(-1)^{n+1} G^{(n+1)}(x)\right]
\end{array}\right)}, \quad m, n \geq 2,1(2)\right\}
\end{gathered}
$$




$$
= \begin{cases}-2 \psi^{\prime}(1), & (m, n)=(0,0) \\
\frac{6 \psi^{\prime \prime}(1)}{1+\psi^{\prime}(1)}, & (m, n) \in\{(1,0),(0,1)\} \\
\frac{12 \psi^{\prime \prime \prime}(1)}{3 \psi^{\prime \prime}(1)-2 \psi^{\prime}(1)}, & (m, n) \in\{(2,0),(0,2)\} \\
-\frac{3 \psi^{\prime \prime \prime}(1)}{\psi^{\prime}(1)}, & (m, n)=(1,1) \\
\frac{10 \psi^{(4)}(1)}{2\left[\psi^{\prime}(1)\right]^{2}-3 \psi^{\prime \prime}(1)}, & (m, n) \in\{(2,1),(1,2)\} \\
\frac{(m+n+2)(m+n+1) \psi^{(m+n+1)}(1)}{\left(\begin{array}{c}
m n\left[(m+1)(n-1) \psi^{(m)}(1) \psi^{(n-1)}(1)\right. \\
\left.+(m-1)(n+1) \psi^{(m-1)}(1) \psi^{(n)}(1)\right]
\end{array}\right)}, & m, n \geq 2\end{cases}
$$

and

$$
\begin{gathered}
\lim _{x \rightarrow \infty} \frac{G^{(m+n+2)}(x)}{G^{(m+1)}(x) G^{(n)}(x)+G^{(m)}(x) G^{(n+1)}(x)} \\
=-\lim _{x \rightarrow \infty} \frac{(-1)^{m+n+2} x^{m+n+3} G^{(m+n+2)}(x)}{\left(\begin{array}{c}
{\left[(-1)^{m+1} x^{m+2} G^{(m+1)}(x)\right]\left[(-1)^{n} x^{n+1} G^{(n)}(x)\right]} \\
+\left[(-1)^{m} x^{m+1} G^{(m)}(x)\right]\left[(-1)^{n+1} x^{n+2} G^{(n+1)}(x)\right]
\end{array}\right)} \\
=-\frac{(m+n+2) ! / 6}{[(m+1) ! / 6](n ! / 6)+(m ! / 6)[(n+1) ! / 6]}=-\frac{6(m+n+1) !}{m ! n !},
\end{gathered}
$$

where we used the limits $(2.1)$ and $(2.2)$ in Lemma 2.4 . It is easy to verify that

$$
\frac{6(m+n+1) !}{m ! n !} \geq \begin{cases}-2 \psi^{\prime}(1), & (m, n)=(0,0) ; \\ \frac{6 \psi^{\prime \prime}(1)}{1+\psi^{\prime}(1)}, & (m, n) \in\{(1,0),(0,1)\} ; \\ \frac{12 \psi^{\prime \prime \prime}(1)}{3 \psi^{\prime \prime}(1)-2 \psi^{\prime}(1)}, & (m, n) \in\{(2,0),(0,2)\} ; \\ -\frac{3 \psi^{\prime \prime \prime}(1)}{\psi^{\prime}(1)}, & (m, n)=(1,1) ; \\ \frac{10 \psi^{(4)}(1)}{2\left[\psi^{\prime}(1)\right]^{2}-3 \psi^{\prime \prime}(1)}, & (m, n) \in\{(2,1),(1,2)\} .\end{cases}
$$

Motivated by these inequalities and other hints, we guess that, for $m, n \in\{0\} \cup \mathbb{N}$, the function

$$
\frac{G^{(m+n+2)}(x)}{G^{(m+1)}(x) G^{(n)}(x)+G^{(m)}(x) G^{(n+1)}(x)}
$$

should be decreasing on $(0, \infty)$.

Remark 5.5. This paper is the seventh one in a series of articles including 4, 5, 6, 7. 8, 10].

\section{REFERENCES}

[1] M. Abramowitz and I. A. Stegun (Eds), Handbook of Mathematical Functions with Formulas, Graphs, and Mathematical Tables, National Bureau of Standards, Applied Mathematics Series 55, Reprint of the 1972 edition, Dover Publications, Inc., New York, 1992.

[2] C. Berg, E. Massa, and A. P. Peron, A family of entire functions connecting the Bessel function $J_{1}$ and the Lambert $W$ function, Constr. Approx. 52 (2020), in press; available online at https://doi.org/10.1007/s00365-020-09499-x 
[3] D. S. Mitrinović, J. E. Pečarić, and A. M. Fink, Classical and New Inequalities in Analysis, Kluwer Academic Publishers, Dordrecht-Boston-London, 1993; available online at https: //doi.org/10.1007/978-94-017-1043-5

[4] F. Qi, Complete monotonicity and monotonicity of two functions defined by two derivatives of a function involving trigamma function, HAL preprint (2020), available online at https: //hal.archives-ouvertes.fr/hal-02993723

[5] F. Qi, Lower bound of sectional curvature of manifold of beta distributions and complete monotonicity of functions involving polygamma functions, MDPI Preprints 2020, 2020110315, 22 pages; available online at https://doi.org/10.20944/preprints202011. $0315 . v 1$.

[6] F. Qi, Monotonicity of a ratio involving trigamma and tetragamma functions, OSF Preprints (2020), available online at https://doi.org/10.31219/osf.io/5rfb8

[7] F. Qi, Necessary and sufficient conditions for two functions defined by two derivatives of a function involving trigamma function to be completely monotonic or monotonic, OSF Preprints (2020), available online at https://doi.org/10.31219/osf.io/6ar4p

[8] F. Qi, Some properties of several functions involving polygamma functions and originating from the sectional curvature of the beta manifold, São Paulo J. Math. Sci. 15 (2021), in press; available online at https://doi.org/10.1007/s40863-020-00193-1

[9] F. Qi and B.-N. Guo, From inequalities involving exponential functions and sums to logarithmically complete monotonicity of ratios of gamma functions, J. Math. Anal. Appl. 493 (2021), no. 1, Article 124478, 19 pages; available online at https://doi.org/10.1016/j. jmaa.2020.124478

[10] F. Qi, L.-X. Han, and H.-P. Yin, Monotonicity and complete monotonicity of two functions defined by three derivatives of a function involving trigamma function, HAL preprint (2020), available online at https://hal.archives-ouvertes.fr/hal-02998203

[11] F. Qi, D.-W. Niu, D. Lim, and B.-N. Guo, Some logarithmically completely monotonic functions and inequalities for multinomial coefficients and multivariate beta functions, Appl. Anal. Discrete Math. 14 (2020), no. 2, 512-527; available online at https://doi.org/10. 2298/AADM191111033Q

[12] R. L. Schilling, R. Song, and Z. Vondraček, Bernstein Functions, 2nd ed., de Gruyter Studies in Mathematics 37, Walter de Gruyter, Berlin, Germany, 2012; available online at https: //doi.org/10.1515/9783110269338

[13] D. V. Widder, The Laplace Transform, Princeton University Press, Princeton, 1946.

[14] Z.-H. Yang and J.-F. Tian, Monotonicity and inequalities for the gamma function, J. Inequal. Appl. 2017, Paper No. 317, 15 pages; available online at https://doi.org/10.1186/ s13660-017-1591-9

[15] Z.-H. Yang, J.-F. Tian, and M.-H. Ha, A new asymptotic expansion of a ratio of two gamma functions and complete monotonicity for its remainder, Proc. Amer. Math. Soc. 148 (2020), no. 5, 2163-2178; available online at https://doi.org/10.1090/proc/14917

Email address: qifeng618@gmail.com, qifeng618@hotmail.com, qifeng618@qq.com $U R L:$ https://qifeng618.wordpress.com, https://orcid.org/0000-0001-6239-2968 